\title{
An Approach for Selection of the Most Desirable Internet Network Based on the Cross-Efficiency Model in Data Envelopment Analysis
}

\author{
Elham ALIPOUR CHAVARI, Mohsen ROSTAMY-MALKHALIFEH*, Farhad HOSEINZADE LOTFI \\ Department of Mathematics, Science and Research Branch, Islamic Azad University, Tehran, Iran \\ elham1446_alipour@yahoo.com,mohsen.rostamy@yahoo.com (*Corresponding author), \\ farhad@hosseinzadeh.ir
}

\begin{abstract}
Choosing an optimized Internet network by the users and providing a desired network by the internet service providers have always been big challenges in this field. Although there are different approaches for selecting the best set of networks such as Analytic Hierarchy Process, Analytic Network Process, and Technique for Order Preference by Similarity to Ideal Solution (TOPSIS), choosing a unique optimal solution still remains an open challenge. The purpose of this paper is to use the decision-making techniques of Data Envelopment Analysis in order to evaluate the existing Internet networks so as to select the most desirable networks. Firstly, a specific Internet network called differentiated service network, that provides the quality of service to the user through the mechanism of Call Admission Control, is stimulated. A novel crossefficiency model is proposed in order to provide a unique ranking of the Internet networks so as to select the optimal network. In particular, secondary goal model based on a satisfaction rate in cross-efficiency is proposed to evaluate and uniquely rank the Internet networks and to select the most desirable network. The present simulated results of 33 networks demonstrate that the proposed model is an effective method for unique ranking of the networks.
\end{abstract}

Keywords: Call Admission Control, Cross-efficiency, Data Envelopment Analysis (DEA), Internet network, Secondary goal.

\section{Introduction}

The volume growth of the services such as Voice over Internet Protocol (VoIP) and video conference made necessary realization of a mechanism to support the quality of service (QoS) for different applications. Various models have been proposed to guarantee the QoS, including the differentiated service (DS) network, presented by IETF (Internet Engineering Task Force). In the architecture of the DS, no Call Admission Control (CAC) mechanism has been considered. To guarantee the QoS, the DS network should support the CAC (Ramirez-Reyna et al., 2018). In order to have the best result of the CAC, some parameters of the network should be considered so that two parameters of loss and delay decrease and at the same time network utilization increases. Thus, different networks can be obtained by changing these parameters.

The question then arises of how to select the most desirable Internet network among the various designed networks. To address this question, multicriteria decision making (MCDM) methods can be used. There are various specialized literatures about MCDM methods. These methods are mathematical techniques used to rank alternatives (Internet networks) based on multiple criteria. Some well-known models in this fields are AHP (Analytic Hierarchy Process), ANP (Analytic Network Process), TOPSIS (Technique for Order of Preference by Similarity to Ideal Solution), and DEA (Data Envelopment Analysis) (Wu et al., 2016).

The present approach to the above question, i.e., how to select the most desirable Internet network, is based on the Data Envelopment Analysis (DEA) (Alipour Chavari \& Rostamy-Malkhalifeh, 2019). DEA is a mathematical technique to evaluate the efficiency of Decision-Making Units (DMUs) with several inputs and outputs, which was presented by Charnes et al. (1987) DEA is used because of three main reasons. First, in DEA, each DMU is evaluated in the best possible condition. So, if a DMU is inefficient, it will not be efficient in any other way (Davtalab-Olyaie, 2019). Second, while MCDM methods require data normalization, DEA can utilize data of inputs and outputs without normalization (Bazrkar et al., 2017). Third, the sources of inefficiency can be analyzed and quantified for every evaluated unit. However, the self-efficiency nature of the DEA is criticized (how it can achieve the efficiency in the best conditions). Therefore, a cross-efficiency evaluation method was introduced in DEA to produce a cross-efficiency matrix (Sexton et al., 1986). But the problem is that there does not exist a unique cross-efficiency matrix. To address this problem, the secondary models were introduced (Emrouznejad \& Liang Yang, 2018). However, they are not able to select a unique optimal network. 
In this paper, a novel cross-efficiency model is devised in order to provide a unique ranking of the Internet networks so as to select the best network. In particular, a secondary goal model, called SRGoal model, is proposed in cross-efficiency to evaluate and rank the Internet networks and to select the most desirable network. The present work is illustrated in summary in Figure 1.

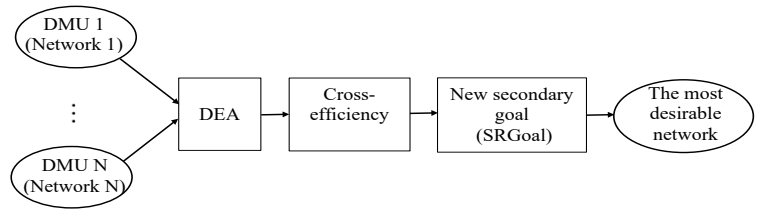

Figure 1. The present scenario

The proposed method is presented in two theoretical and practical parts. In the first part, a secondary goal is presented regarding the multi-objective programming and goal programming (Ehrgott, 2002). In the second part, Internet networks are stimulated using NS-2 simulator (Zhou et al., 2018), and evaluated using a cross-efficiency model. The simulated results show that the proposed method can uniquely rank the Internet networks and select the most desirable network.

The main contributionsof this paper are:

- a novel method, called SRGoal, is proposed to extract a secondary goal in the crossefficiency model;

- the practicality of this method for unique ranking and for the selection of the best Internet networks by simulation is shown.

As far as anyone knows, SRGoal is the first model that uses a secondary goal to uniquely rank Internet networks.

The paper is organized as follows. Section 2 introduces background concepts and literature reviews. Section 3 discusses Call Admission Control criteria. Section 4 presents the secondary goal model in cross-efficiency and the present proposed approach. Section 5 reports the present simulated scenario in order to select the most desirable Internet network by the present proposed model. Section 6 provides a discussion regarding the results of the experiment. Finally, section 7 offers the conclusion of the paper and some prospects for the development of the proposed research in future works.

\section{Background and Literature Review}

The work presented in this paper has two main steps. In the first step, the CAC is added to the DS network and, in the second step, the different networks are uniquely ranked, and the optimal network is selected. In the first step, in order to ensure the QoS guarantee, the DS network should support the admission control mechanism (Chen et al., 2019). The process of deciding to accept or reject a new request is called admission control. The proper admission control algorithm has a significant impact on network efficiency. An algorithm which unnecessarily rejects flows leads to an under-utilization network. Similarly, an algorithm which incorrectly accepts many flows results in a violation from QoS guarantee (Alipour \& Mohammadi, 2008).

Three main categories of CAC have been proposed in the specialized literatures. ParameterBased Admission Control (PBAC), MeasurementBased Admission Control (MBAC) and EndpointBased Admission Control (EBAC) (Alipour \& Mohammadi, 2008). PBAC is based only on some traffic descriptors such as the peak and average rate. In MBAC, some real-time measuring techniques are used to measure the required traffic parameters. In EBAC, before the traffic flow is established, some probing packets are sent. The end-to-end path should be the same for both probing packets and traffic flows. The problem of EBAC method is that the setup delays may be high. In addition, simultaneous probing of many different sources can take more bandwidth and degrade the utilization of the network. In this paper, the PBAC approach is used. By PBAC, the limitations of real-time measuring and monitoring of traffic in MBAC can be avoided (especially in high-speed networks). In addition, due to PBAC simplicity and ease of implementation, it is preferred. In PBAC, the admission control assumes that complete information is available from all the traffic sources which can be used in any link. It is also assumed that the current number of created services is specified. This information enables the admission control routine to calculate the required bandwidth.

In the second step of our work, DEA technique is used to select the most desirable Internet network. There are exhaustive literatures for MCDM

https://www.sic.ici.ro 
methods, such as AHP, ANP, and TOPSIS (Oukil $\&$ Amin, 2015). AHP is a structured technique to organize and analyze complex decision. However, AHP is unable to apply a pairwise comparison between simulated networks. In addition, the underlying mathematics of AHP is complicated (Wu et al., 2016). ANP is an extended process of AHP used in multi-criteria decision analysis. ANP structures a decision problem as a network, instead of hierarchy, and it suffers from the same drawbacks of AHP. TOPSIS is also an MCDM method. It ranks alternatives by construction of a decision matrix (Moeini et al., 2015). TOPSIS calculates the separation distance of each competitive alternative from the ideal and non-ideal solution, and it ranks the alternatives based on closeness of each alternative to the ideal solution. However, TOPSIS requires a big decision matrix for calculation that makes it computationally very expensive (Ho et al., 2018).

DEA technique is based on a mathematical model used to evaluate the efficiency of Decision-Making Units. Because of the reasons, mentioned in Introduction, this technique is used in the preset work. Over the past 40 years, many studies have been done in this field (Hosseinzadeh Lotfi et al., 2007). However, the self-efficiency nature of the DEA is criticized (efficiency in the best conditions). Therefore, a cross-efficiency evaluation method was introduced in DEA. Cross-efficiency is an extended method in DEA for DMUs ranking, proposed by Sexton et al. (1986). In this method, the efficiency of the other units is calculated by the obtained weights from the evaluation of $\mathrm{DMU}_{j}(j=1, \ldots, n)$ and a matrix known as the cross-efficiency matrix is produced. Finally, DMUs are ranked by obtaining the cross-efficiency score from this matrix (Sexton et al., 1986).

DEA has been successfully used in real-world problems (Ang et al., 2018). The authors evaluated the performance of Taiwan hotels using the concept of group cross-efficiency. Chiang Lee (2019) evaluated the commercial banks in Taiwan using the combination model of the crossefficiency and the entropy Shannon. Chen et al. (2019) evaluated the meta-frontier using a crossefficiency model to evaluate performance.

While the basic models of DEA have multiple optimal solutions, the obtained cross-efficiency matrix is different regarding the type of the used software. The primary problem of this model is the failure to provide a cross-efficiency matrix because of the multiple optimal solutions in the traditional DEA models. The authors in (Rodder \& Reucher, 2001) proposed a complementary model to address this problem, which was later introduced as the secondary goal model. Several researchers have proposed the secondary goal models in the field of cross efficiency (Emrouznejad \& Liang Yang, 2018). For example, for the first time, the secondary optimistic and pessimistic models regarding the maximum and minimum efficiency of the other DMUs, respectively, were proposed. In addition, Liang et al. (2008) proposed a secondary goal model using the concepts of deviation from the efficiency, and Ramirez-Reyna et al. (2018) proposed another secondary goal method and transformed it into a linear programming model using multi-objective methods.

Although there are several studies about the secondary goal method, they are not able to uniquely rank the DMUs. The question here is how to choose the best internet network using the Data Envelopment Analysis models. Recently, Alipour Chavari \& Rostamy-Malkhalifeh (2019) selected the best-designed internet network using the basic models of Data Envelopment Analysis. However, efficiency evaluation by the basic models of Data Envelopment Analysis does not usually lead to a unique ranking. The aim of this work is to propose a secondary goal model based on a newly introduced concept of satisfaction rate so that it can select a unique desirable Internet network. Due to the different powers of the secondary goal models in ranking, they can be used to solve the problems of the existing models in this field.

\section{Admission Control Criteria}

Different networks have been presented to guarantee the QoS among which DS is the most popular. DS includes two main classes of services. The first main class is expedited forwarding (EF) class which offers services that are extremely sensitive to delay and bandwidth such as VOIP and videoconference. The other class is assured forwarding (AF) class that has been designed to ensure that packets are forwarded with a high probability of delivery as long as the aggregate traffic in a forwarding class does not exceed the subscribed information rate.

Admission criteria are the rules by which an admission control scheme accepts or rejects 
a request (Zanuttini et al., 2018). Different admission control criteria have been proposed. In this paper, the equivalent capacity $\mathrm{C}_{\text {est }}$ is used (Karimi et al., 2018), since this criterion is based on PBAC mechanism. PBAC is used because of its ease of implementation. In addition, this work focuses on improving the unique selection of an Internet network by DEA model, not on improving the Call Admission Control methods. In order to improve the quality of network service, the PBAC mechanism is added to the edge routers of the network. It is assumed that through the provision of the network and traffic engineering, the minimum total bandwidth of $\mathrm{C}$ total is available end-to-end. The other assumption is that whenever a source wants to be active and sends traffic, it will inform its request to the ingress node through a reservation protocol.

In the PBAC mechanism, having the number of the active sources and the peak rate of the new traffic source $\mathrm{P}_{\text {new }}$ and also assuming that the new source is sending traffic with its peak rate, the required bandwidth for accepting the new request is computed according to (1):

$c_{\text {est }}=\sum_{i=1}^{n} p_{i}+p_{\text {new }}$

where $p_{i}$ is the pick sending rate of the active sources, and the parameter $n$ is the number of active sources, which is available in the network. Therefore, it is possible to estimate the required bandwidth to receive the new traffic according to $c_{\text {est }}$ such that this estimation is used in the admission control criterion.

By having $c_{\text {total }}$ and measuring $c_{\text {est }}$, the admission control criterion is presented according to (2).

$\begin{array}{lll}\text { if } & c_{\text {est }} \leq c_{\text {total }} & \text { admit } \\ \text { if } & c_{\text {est }}>c_{\text {total }} & \text { reject }\end{array}$

This scheme guarantees the QoS. Even if all the sources send traffic with the peak rate, the network will be able to deliver the desired QoS.

\section{Cross-efficiency}

One of the methods of ranking the efficient Decision-Making Units in DEA is cross-efficiency model which has a high ability to differentiate efficient units (Moeini et al., 2015). The efficiency in DEA is calculated by the ratio of "balanced outputs" to "balanced inputs". The selection of weights in the DEA linear programming model is in such a way that it allows the unit under investigation to maximize the amount of its efficiency over the other units.

The main idea of cross-efficiency is to use the DEA as a reference assessment rather than a pure evaluation model (self-assessment). The two main advantages of cross-efficiency evaluation are as follows:

1. Cross-efficiency provides an efficiency ranking among all the Decision-Making Units (DMUs) to distinguish between the best and the worst performances;

2. Cross-efficiency can eliminate the need for the weight limits for a variety of applications and thus it violates the DEA's unrealistic weighting method (Anderson et al., 2002; Lotfi \& Rostamy-MaleKhalifeh, 2016).

In DEA, the following classic notations are used: Suppose there is a set of DMUs. Each DMU has $m$ distinct inputs, given as $x_{i j}(i=1, \ldots, m)$ and $s$ distinct outputs, given as $y_{r j}(r=1, \ldots, s)$.

The cross-efficiency of the DEA is a traditional development in the two-step process. More precisely, in step 1, the self-evaluation efficiency of each DMU is calculated based on the DEA model of Copper Charnes Rhodes (CCR) (Karimi et al., 2018). In step 2, the weights obtained from step 1 are applied to all the other DMUs in order to achieve a score which is referred to as the crossefficiency evaluation score (Karimi et al., 2018). In the following subsection, the cross-efficiency model is presented in 4 steps.

\subsection{The Cross-efficiency Model}

1. Self-evaluation efficiency of $\mathrm{DMU}_{\mathrm{d}}$ using the CCR model in DEA is calculated as follows:

$$
\begin{aligned}
& E_{d d}=\operatorname{Max} \sum_{r=1}^{s} u_{r d} y_{r d} \\
& \text { s.t. } \quad \sum_{r=1}^{s} u_{r d} y_{r j}-\sum_{i=1}^{m} v_{i d} x_{i j} \leq 0 \quad j=1, \ldots, n \\
& \sum_{i=1}^{m} v_{i d} x_{i d}=1 \\
& v_{i d}, u_{r d} \geq 0 \quad r=1, \ldots, s, \quad i=1, \ldots, m
\end{aligned}
$$

where $u_{r d}$ and $v_{i d}$ are the weights of the output $r$ and the input $\mathrm{i}$, respectively. For each $D M U_{d}$, a 
set of the optimal weights of $v_{1 d}^{*}, v_{2 d}^{*}, \ldots, v_{m d}^{*}$ and $u_{1 d}^{*}, u_{2 d}^{*}, \ldots, u_{s d}^{*}$ is obtained by solving the model of step 1 .

2. Cross-Efficiency from each $D M U$, using optimal weights $D M U_{d}$ i.e. $E_{d j}$ is calculated as follows:

$$
E_{d j}=\frac{\sum_{r=1}^{s} u_{r d}^{*} y_{r j}}{\sum_{i=1}^{m} v_{i d}^{*} x_{i j}} \quad j=1, \ldots, n ; j \neq d
$$

Therefore, the Cross-Efficiency Matrix (CEM) is constructed using Equation (4).

3. In the Cross-Efficiency Matrix, for each $\operatorname{DMU}_{j}(j=1, \ldots, n)$ in the column $\mathrm{J}$, the average of all $E_{d j}(d=1, \ldots, n)$ is calculated according to Equation (5), and this average is considered the cross-efficiency score for $\mathrm{DMU}_{j}$.

$$
\bar{E}_{j}=\frac{1}{n} \sum_{d=1}^{n} E_{d j}
$$

Cross-efficiency score can also be referred to as a cumulative score with equal weight of $1 / n$.

4. The decision units are ranked using the crossefficiency score.

\subsection{The Proposed Cross-efficiency Approach Based on Satisfaction Ratio}

This subsection introduces a new concept, namely the satisfaction ratio, and presents a secondary goal model for cross-efficiency.

The model (6) calculates an ideal value of efficiency for $\operatorname{DMU}_{j}(j=1, \ldots, n ; j \neq d)$ according to the weights of $\mathrm{DMU}_{d}$.

$$
\begin{aligned}
& D M U_{d} \\
& \bar{E}_{d j}=\operatorname{Max} \sum_{r=1}^{s} u_{r d} y_{r j} \\
& \text { s.t. } \sum_{i=1}^{m} v_{i d} x_{i j}=1 \quad j=1, \ldots, n \\
& \sum_{r=1}^{s} u_{r d} y_{r d}-E_{d d}^{*} \sum_{i=1}^{m} v_{i d} x_{i d}=0 \\
& \sum_{r=1}^{s} u_{r d} y_{r j}-\sum_{i=1}^{m} v_{i d} x_{i j} \leq 0 \quad j=1, \ldots, n \\
& u_{r d} \geq 0, v_{i d} \geq 0 \quad i=1, \ldots, m, r=1, \ldots, s
\end{aligned}
$$

where $E_{d d}^{*}$ is the efficiency value of $\mathrm{DMU}_{d}$ using model (3). Similarly, one can set an ideal efficiency value for $\operatorname{DMU}_{j}(j=1, \ldots, n ; j \neq d)$ that can be calculated according to the weights of $\mathrm{DMU}_{d}$ as follows:

$$
\begin{array}{rll}
\underline{E}_{d j}= & \operatorname{Min} \sum_{r=1}^{s} u_{r d} y_{r j} & \\
\text { s.t. } & \sum_{i=1}^{m} v_{i d} x_{i j}=1 & j=1, \ldots, n \\
& \sum_{r=1}^{s} u_{r d} y_{r d}-E_{d d}^{*} \sum_{i=1}^{m} v_{i d} x_{i d}=0 & \\
& \sum_{r=1}^{s} u_{r d} y_{r j}-\sum_{i=1}^{m} v_{i d} x_{i j} \leq 0 & j=1, \ldots, n \\
& u_{r d} \geq 0, v_{i d} \geq 0 & i=1, \ldots, m, r=1, \ldots, s
\end{array}
$$

$\mathrm{DMU}_{j}(j=1, \ldots, n ; j \neq d)$ is always trying to derive the efficiency value of $\bar{E}_{d j}$. However, deriving this value for all $\operatorname{DMU}_{j}(j=1, \ldots, n ; j \neq d)$ is impossible. $\operatorname{DMU}_{j}(j=1, \ldots, n ; j \neq d)$ also tries to avoid the efficiency value of $\underline{E}_{d j}$. Based on these explanations, the following concept can be provided.

Definition. For $\operatorname{DMU}_{j}(j=1, \ldots, n ; j \neq d)$ the satisfaction ratio according to the weights of $D M U_{d}$ is defined as follows:

$\varphi_{j}=\frac{1}{\bar{E}_{d j}-\underline{E}_{d j}}\left(w_{1}\left(E_{d j}-\underline{E}_{d j}\right)-w_{2}\left(\bar{E}_{d j}-E_{d j}\right)\right)$

Here, we call $\varphi_{j}$ the ratio of satisfaction, in which the higher the value, the greater the satisfaction. $w_{1}$ and $w_{2}$ are the weights taken by the decision maker in order to assess the efficiency gap between its ideal or anti-ideal. Also, $w_{1}+w_{2}=1$. $E_{d j}$ is the calculated efficiency value for $D M U_{j}$ according to the weights of $D M U_{d}$ by the model. Here, according to the satisfaction ratio defined above, the following model is proposed to derive the efficiency of $\operatorname{DMU}_{j}(j=1, \ldots, n ; j \neq d)$ using the optimal weights of $\mathrm{DMU}_{d}$.

$$
\begin{array}{ll}
\operatorname{cax}_{\substack{1 \leq j \leq n \\
j \neq d}} & \varphi_{j}=\frac{1}{\bar{E}_{d j}-\underline{E}_{d j}}\left(w_{1}\left(E_{d j}-\underline{E}_{d j}\right)-w_{2}\left(\bar{E}_{d j}-E_{d j}\right)\right) \\
\text { s.t. } \quad & \sum_{i=1}^{m} \sum_{j=1}^{n} v_{i d} x_{i j}=1 \\
& \sum_{r=1}^{s} u_{r d} y_{r d}-E_{d d}^{*} \sum_{i=1}^{m} v_{i d} x_{i d}=0 \\
& \sum_{r=1}^{s} u_{r d} y_{r j}-E_{d j} \sum_{i=1}^{m} v_{i d} x_{i j}=0 \quad j=1, \ldots, n \\
& \sum_{r=1}^{s} u_{r d} y_{r j}-\sum_{i=1}^{m} v_{i d} x_{i j} \leq 0 \quad j=1, \ldots, n \\
& u_{r d} \geq 0, v_{i d} \geq 0 \quad i=1, \ldots, m \quad, r=1, \ldots, s
\end{array}
$$

where the ideal values $\bar{E}_{d j}$ and the anti-ideal values $\underline{E}_{d j}$ are derived using models (6) and (7), respectively. Also $E_{d j}$ is the efficiency variable 
of $\mathrm{DMU}_{j}$ using the optimal weights of $\mathrm{DMU}_{d}$, which is determined by the model. Here, the purpose is to derive the maximum satisfaction ratio for $\operatorname{DMU}_{j}(j=1, \ldots, n ; j \neq d)$, so that the efficiency of $\mathrm{DMU}_{d}$ remains constant.

The above model is a multi-objective model. But using multi-objective methods, it can be transformed into a single-objective programming model and then it can be solved (Ehrgott, 2000). The weighted method is one of the methods used in this paper. Therefore, by using the weighted method, model (9) is converted to the following single-objective model:

$$
\begin{aligned}
& \operatorname{Max} \sum_{\substack{j=1 \\
j \neq d}}^{s} W_{j}^{\prime} \varphi_{j} \\
& \text { s.t. } \quad \varphi_{j}=\frac{1}{\bar{E}_{d j}-\underline{E}_{d j}}\left(w_{1}\left(E_{d j}-\underline{E}_{d j}\right)-w_{2}\left(\bar{E}_{d j}-E_{d j}\right)\right), 1 \leq j \leq n ; j \neq d \\
& \sum_{i=1}^{m} \sum_{j=1}^{n} v_{i d} x_{i j}=1 \\
& \sum_{r=1}^{s} u_{r d} y_{r d}-E_{d d}^{*} \sum_{i=1}^{m} v_{i d} x_{i d}=0 \\
& \sum_{r=1}^{s} u_{r d} y_{r j}-E_{d j} \sum_{i=1}^{m} v_{i d} x_{i j}=0 \quad j=1, \ldots, n \\
& \sum_{r=1}^{s} u_{r d} y_{r j}-\sum_{i=1}^{m} v_{i d} x_{i j} \leq 0 \quad j=1, \ldots, n \\
& \sum_{\substack{j=1 \\
j \neq d}}^{s} W_{j}^{\prime}=1 \\
& u_{r d} \geq 0, v_{i d} \geq 0 \quad i=1, \ldots, m, r=1, \ldots, s
\end{aligned}
$$

where $W_{j}^{\prime}$ is the weight related to the objective $j$ in the weighted method, which is determined by the decision maker, but these weights should be non-negative $\sum_{\substack{j=1 \\ j \neq d}}^{s} W_{j}^{\prime}=1$. Note that if $\underline{E}_{d j}=\bar{E}_{d j}$ (For $d=1, \ldots, n ; j=1, \ldots, n)$, the satisfaction ratio will be 1 in the fraction. The output of this model is the satisfaction ratio $\varphi_{j}$ and the efficiency value $E_{d j}(j=1, \ldots, n ; j \neq d)$, which can be placed in the row $d$ of the new crossefficiency matrix. Likewise, the cross-efficiency matrix of the above model is constructed by solving $n$ times, and the cross-efficiency score is calculated as follows:

$$
E_{d}=\frac{\sum_{j=1}^{n} E_{d j}}{n}
$$

Given the above formula, we have the following algorithm for ranking DMUs with respect to the satisfaction ratio:

\subsection{The Final Proposed SRGoal Model}

1. The efficiency values $E_{d d}$ for $(d=1, \ldots, n)$ are calculated by using model (3);

2. The ideal values $\bar{E}_{d j}$ and anti-ideal values $\underline{E}_{d j}$ are calculated using models (6) and (7), respectively;

3. The values $E_{d j}(j=1, \ldots, n ; j \neq d)$ are calculated using model (10) and the crossefficiency matrix is formed;

4. The cross-efficiency score is calculated using Equation (11);

5. The Decision-Making Units are ranked based on the cross-efficiency score.

All the three models (3), (6), and (7) as well as (10) are the type of the mathematical programming and always have the finite optimum value. In addition, $E_{d j}(j=1, \ldots, n)$ derived for model (3) is always between zero and one. Accordingly, the cross-efficiency score derived using (11) is always between zero and one. The higher the cross-efficiency score, the better the rating of the unit under evaluation.

\section{Evaluation and Selection of the Most Desirable Internet Network}

\subsection{Network Topology}

The NS-2 simulator is used to evaluate the efficiency of the admission control algorithm in the differentiated service network (Zhou et al., 2018). S. Andreozzi's patch model was used to support the basic Differ functionalities. The dumbbell topology is used, as shown in Figure 2 (Alipour \& Mohammadi, 2008). This network is considered as a DMU, and different DMUs would be made by specifying different inputs, according to Table 1. All simulations and programs were run on a $2.6 \mathrm{GHz} \mathrm{CPU}$ with $16 \mathrm{~GB}$ of memory running Windows 7. Simulation with 10 different seed values is run in each simulated case. Simulated time for each scenario is 1000 seconds.

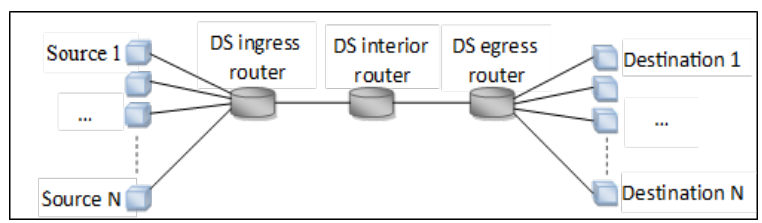

Figure 2. Network topology (Alipour \& Mohammadi, 2008) 
In this paper, the simulation of the two classes of network traffic has been considered: the Expedited Forwarding (EF) class and the Best-Effort (BE) class. 120 sources in the desirable topology are considered that generate traffic in the network. These sources generate two types of traffic: EF traffic, which is the VoIP traffic, and BE traffic, which produces a high percentage of Internet traffic. The details of simulated traffic, inputs and outputs are represented in the next subsection.

\subsection{Definition of Inputs and Outputs}

The first input (I1): among all the sources, the sources which deal with the production of the EF traffic are considered the first input. The values of these inputs are shown in Table 1. The number of BE sources is also changed which is equal to the difference between the number of the EF sources and the number of all the sources. For these two traffic classes, the separate queues are used.

The second input (I2): the size of EF traffic queue is considered the second input which is shown in Table 1. Token bucket meter is used for classifying the packets in EF traffic queue. The size of BE traffic queue is considered to be 20. For modeling the VoIP traffic, the exponential ON/OFF model with a peak rate of $64 \mathrm{Kbps}$ is specified, and the mean durations used for the $\mathrm{ON}$ and $\mathrm{OFF}$ periods are 1.004 second $1.587 \mathrm{sec}$, respectively. BE traffic is a type of traffic with Constant Bit Rate (CBR) of $50 \mathrm{Kbps}$ and a packet size of 1000 bytes. The duration of the source traffic is considered a random number with a normal distribution and a mean of 10 seconds. The capacity of the output link is 3.1 Mbps which is divided between the EF and BE traffic.

The third input (I3): the amount of the link capacity allocated to EF traffic is considered the third input as shown in Table 1.

The desirable outputs are achieved by performing the simulations for each DMU with the duration of 1000 seconds.

The first output (O1) represents the packet loss rate related to EF traffic.

The second output $(\mathrm{O} 2)$ represents the delay of the $\mathrm{EF}$ traffic packets from the source to the destination.

The third output (O3) represents the network utilization.
Table 1. DMUs and the input and output values

\begin{tabular}{|c|c|c|c|c|c|c|}
\hline DMU & I1 & I2 & I3 & 01 & $\mathbf{O 2}$ & $\mathbf{O 3}$ \\
\hline DMU1 & 90 & 3 & 600 & 83.46 & 5.37 & 13.94 \\
\hline DMU2 & 70 & 3 & 600 & 84.62 & 4.28 & 13.52 \\
\hline DMU3 & 50 & 3 & 600 & 85.73 & 3.89 & 13.60 \\
\hline DMU4 & 30 & 3 & 600 & 85.02 & 4.19 & 13.94 \\
\hline DMU5 & 90 & 4 & 500 & 85.85 & 6.19 & 15.37 \\
\hline DMU6 & 70 & 4 & 500 & 87.50 & 5.28 & 14.68 \\
\hline DMU7 & 50 & 4 & 500 & 82.32 & 3.35 & 14.35 \\
\hline DMU8 & 30 & 4 & 500 & 84.36 & 5.65 & 15.25 \\
\hline DMU9 & 50 & 3 & 500 & 85.49 & 6.97 & 14.00 \\
\hline DMU10 & 50 & 3 & 500 & 84.47 & 6.75 & 14.93 \\
\hline DMU11 & 30 & 5 & 500 & 84.88 & 5.42 & 15.82 \\
\hline DMU12 & 50 & 5 & 500 & 84.67 & 2.85 & 14.46 \\
\hline DMU13 & 70 & 5 & 500 & 86.05 & 3.87 & 15.24 \\
\hline DMU14 & 30 & 4 & 700 & 79.85 & 0.12 & 13.55 \\
\hline DMU15 & 30 & 3 & 700 & 78.00 & 2.45 & 13.40 \\
\hline DMU16 & 50 & 3 & 700 & 78.50 & 2.09 & 12.97 \\
\hline DMU17 & 70 & 3 & 700 & 76.91 & 2.22 & 13.07 \\
\hline DMU18 & 70 & 4 & 700 & 78.70 & 0.10 & 13.27 \\
\hline DMU19 & 60 & 3 & 500 & 86.72 & 5.54 & 13.27 \\
\hline DMU20 & 90 & 4 & 700 & 78.66 & 0.08 & 13.35 \\
\hline DMU21 & 90 & 5 & 600 & 88.16 & 1.35 & 14.75 \\
\hline DMU22 & 70 & 5 & 600 & 55.42 & 0.63 & 14.23 \\
\hline DMU23 & 30 & 5 & 600 & 88.54 & 1.05 & 14.83 \\
\hline DMU24 & 50 & 5 & 600 & 88.72 & 0.39 & 13.96 \\
\hline DMU25 & 70 & 2 & 600 & 77.56 & 12.35 & 13.00 \\
\hline DMU26 & 90 & 3 & 700 & 72.39 & 5.70 & 12.52 \\
\hline DMU27 & 90 & 3 & 500 & 82.89 & 9.30 & 14.46 \\
\hline DMU28 & 90 & 5 & 500 & 87.11 & 6.31 & 16.48 \\
\hline DMU29 & 50 & 4 & 700 & 80.07 & 0.13 & 13.06 \\
\hline DMU30 & 90 & 4 & 600 & 86.54 & 2.12 & 14.60 \\
\hline DMU31 & 70 & 3 & 600 & 87.21 & 6.31 & 14.19 \\
\hline DMU32 & 30 & 4 & 600 & 87.82 & 1.62 & 14.58 \\
\hline DMU33 & 50 & 4 & 600 & 88.07 & 1.10 & 13.79 \\
\hline
\end{tabular}

\subsection{Simulation Results}

This subsection evaluates the simulated results of our proposed SRGoal model and compare the results with the ones of the CCR model. In the 
first step, CCR model is used in (3) and the selfevaluation efficiency is derived. The results of the implementation of the model are presented in Table 2. Please note that all the linear programming models were executed using Lingo 11 software.

Table 2. Results of self-evaluation using CCR model

\begin{tabular}{|c|c|}
\hline DMU & Efficiency Score \\
\hline DMU1 & 0.9 \\
\hline DMU2 & 0.9 \\
\hline DMU3 & 0.94 \\
\hline DMU4 & 1 \\
\hline DMU5 & 1 \\
\hline DMU6 & 0.96 \\
\hline DMU7 & 0.95 \\
\hline DMU8 & 1 \\
\hline DMU9 & 1 \\
\hline DMU10 & 1 \\
\hline DMU11 & 1 \\
\hline DMU12 & 0.98 \\
\hline DMU13 & 0.98 \\
\hline DMU14 & 0.91 \\
\hline DMU15 & 0.93 \\
\hline DMU16 & 0.84 \\
\hline DMU17 & 0.77 \\
\hline DMU18 & 0.68 \\
\hline DMU19 & 1 \\
\hline DMU20 & 0.68 \\
\hline DMU21 & 0.83 \\
\hline DMU22 & 0.75 \\
\hline DMU23 & 1 \\
\hline DMU24 & 0.86 \\
\hline DMU25 & 0.78 \\
\hline DMU26 & 1 \\
\hline DMU27 & 1 \\
\hline DMU28 & 1 \\
\hline DMU29 & 0.7 \\
\hline DMU30 & 0.84 \\
\hline DMU31 & 1 \\
\hline DMU32 & 1 \\
\hline DMU33 & 0.86 \\
\hline
\end{tabular}

As it can be seen from Table 2, out of 33 DMUs, 12 units received the efficiency score of 1 and they are efficient. The rest of the DMUs are inefficient. The question that arises here is which DMU is the most desirable network. Here, according to the results of model (3), all 12 efficient DMUs represent optimal choices for an Internet network and no unique choice can be made. The proposed algorithm for the unique choice is presented in the following.
First the ideal values $\bar{E}_{d j}$ and the anti-ideal values $\underline{E}_{d j}(j=1, \ldots, n ; j \neq d, d=1, \ldots, n)$ are calculated from the models (6) and (7), respectively. The tables of these matrixes are also obtained. In the next step, the values of $E_{d j}$ from the model (10) are calculated, together with the cross-efficiency matrix. Here, the weights of $W_{j}^{\prime}$ are also considered the same (1/33). At this stage, the column average is used and the cross-efficiency score is derived, as presented in Table 3.

Table 3. The cross-efficiency score based on SRGoal

\begin{tabular}{|c|c|c|}
\hline DMU & Rank & Cross-efficiency score \\
\hline DMU1 & 0.677576 & 10 \\
\hline DMU2 & 0.570303 & 26 \\
\hline DMU3 & 0.522424 & 30 \\
\hline DMU4 & 0.681212 & 9 \\
\hline DMU5 & 0.654545 & 14 \\
\hline DMU6 & 0.62303 & 21 \\
\hline DMU7 & 0.609091 & 22 \\
\hline DMU8 & 0.538485 & 27 \\
\hline DMU9 & 0.629697 & 19 \\
\hline DMU10 & 0.787273 & 1 \\
\hline DMU11 & 0.699394 & 6 \\
\hline DMU12 & 0.625758 & 20 \\
\hline DMU13 & 0.586667 & 25 \\
\hline DMU14 & 0.647879 & 16 \\
\hline DMU15 & 0.654242 & 15 \\
\hline DMU16 & 0.684242 & 8 \\
\hline DMU17 & 0.675758 & 11 \\
\hline DMU18 & 0.536061 & 28 \\
\hline DMU19 & 0.734818 & 2 \\
\hline DMU20 & 0.727273 & 5 \\
\hline DMU21 & 0.590303 & 24 \\
\hline DMU22 & 0.659394 & 13 \\
\hline DMU23 & 0.52697 & 29 \\
\hline DMU24 & 0.502727 & 32 \\
\hline DMU25 & 0.513636 & 31 \\
\hline DMU26 & 0.453333 & 33 \\
\hline DMU27 & 0.671818 & 12 \\
\hline DMU28 & 0.694848 & 7 \\
\hline DMU29 & 0.646667 & 17 \\
\hline DMU30 & 0.597576 & 23 \\
\hline DMU31 & 0.73303 & 3 \\
\hline DMU32 & 0.73097 & 4 \\
\hline DMU33 & 0.636061 & 18 \\
\hline
\end{tabular}

The rank of the Internet networks has been shown in the third column of Table (3). As is can be seen, the proposed algorithm provides a unique ranking of Internet networks such that the networks of 
10,32 , and 31 obtain the first to the third ranks, respectively. So, they can be good choices for the selection of the most desirable network.

According to the presented algorithmic results in this paper, the network 10 is the optimal choice among the designed networks. By comparing the inputs and outputs of this network, the feature of a good network is that it can produce the outputs $80.47,6.75$, and 13.93 by the inputs 70,3 , and 500 , respectively.

\section{Discussion}

In this paper, the main goal is to select the most desirable Internet network using cross-efficiency. For this purpose, 33 networks were simulated. The results of the proposed model showed that the $10^{\text {th }}$, the $19^{\text {th }}$ and the $31^{\text {th }}$ networks obtained the first three ranks, and are more desirable than the other networks. These three networks are displayed in Table 4.

Table 4. The input and output values for most desirable networks by SRGoal model

\begin{tabular}{|c|c|c|c|c|c|c|}
\hline DMU & I1 & I2 & I3 & O1 & O2 & O3 \\
\hline DMU10 & 50 & 3 & 500 & 84.47 & 6.75 & 14.93 \\
\hline DMU19 & 60 & 3 & 500 & 86.72 & 5.54 & 13.27 \\
\hline DMU31 & 70 & 3 & 600 & 87.21 & 6.31 & 14.19 \\
\hline
\end{tabular}

These three networks can provide a solution for selecting the best Internet network. It needs to be emphasized that in MCDM methods, in particular in DEA, the best alternatives (Internet networks) are selected based on all the criteria, and it is possible for the best alternatives to be powerful in several criteria, but weak in one or in more than one criterion, in comparison with the other alternatives. For example, in Table 4, the network of 10, which has the first rank, performs well for the second and the third outputs compared to the networks of 19 and 31. However, in terms of the first output (84.47), it performs worse than the two other networks. The solution proposed for this problem is to use the average of each input and the average of each output of these three networks as a measure for selecting the best network. The final network that was selected as the desirable one is presented in Table 5. In this table, for example, the third input is the average of three inputs in the networks of $10,19,31$, which is 533.3. As a result, the most desirable network should have the following parameters:
Table 5. The most desirable network

\begin{tabular}{|c|c|c|c|c|c|c|}
\hline DMU & I1 & I2 & I3 & O1 & O2 & O3 \\
\hline DMU $_{d}$ & 60 & 3 & 533.3 & 86.13 & 6.2 & 14.13 \\
\hline
\end{tabular}

Furthermore, in the proposed method, if two networks of 10 and 26 (the first rank and the last rank, respectively) are compared, it can be observed that, although the inputs criteria in the network of 10 always require lower input values when compared to the network of 26 , the output criteria in the network of 10 always produce higher outputs when compared to the network of 26. This situation it leads to the conclusion that the network of 10 has a much better rank in comparison with network of 26 .

\section{Conclusion}

Although QoS of the Internet networks has not received much attention in Iran so far, it is foreseen that it will become more important in the near future for two reasons. The first reason is the emergence of Next Generation Networks (NGNs) based on IP, which will bring a variety of services to be used in the commercial sector in a manner in which the customers of these services are less likely to be harmed by the service providers. The second reason which is relevant to the first one is the privatization and liberalization process that is hoped to provide a competitive market in the country. In such a market, the service providers who can be able to deliver the QoS guarantee to the customers will stay in competition. Differentiated service architecture is a technology that can improve the QoS even for the real-time services. The Call Admission Control is needed to achieve the scalability and efficiency simultaneously.

In this paper, the Internet network traffic was simulated by using a NS-2 simulator. In the first step, the admission control mechanism was added to the edge routers of the DS network. Then, in the next step, the network's inputs were changed, including the buffer size, the number of the input sources and the link bandwidth, and the outputs of the network, which are loss, delay, and utilization, were extracted. The present work proposes an efficient method for the optimal design of the network to achieve the highest efficiency. The DS network was selected as DMU and by giving different inputs, different DMUs were made. Then, the efficiency of these inputs was investigated by DEA model. It also provided a unique ranking of Internet networks to select the most desirable network. 


\section{REFERENCES}

Alipour Chavari, E. \& Rostamy-Malkhalifeh, M. (2019). Internet network design for quality of service guarantee using Data Envelopment Analysis (DEA), International Journal of Data Envelopment analysis, $7(2), 1-14$.

Alipour, E. \& Mohammadi, K. (2008). Adaptive admission control for quality of service guarantee in differentiated services networks, International Journal of Computer Science and Network Security, 8(6), 93-98.

Anderson, T. R., Hollings Worth, K. B. \& Inman, L. B. (2002). The fixed weighting nature of a cross evaluation model, Journal of Productivity Analysis, 17, 249-255.

Ang, S., Chen, M. \& Yang, F. (2018). Group crossefficiency evaluation in data envelopment analysis: An application to Taiwan hotels, Computers \& Industrial Engineering, 125, 190-199.

Bazrkar, A., Iranzadeh, S. \& Feghhi Farahmand, N. (2017). Total quality model for aligning organization strategy, improving performance, and improving customer satisfaction by using an approach based on combination of balanced scorecard and lean six sigma, Cogent Business \& Management, 4(1), 1-16.

Charnes, A., Cooper, W. W. \& Rhodes, E. (1978). Measuring the efficiency of decision making units, European Journal of Operational Research, 2, 429-444.

Chen, L., Huang, H., Li, M. J. \& Wang, Y. M. (2019). Meta-frontier analysis using cross-efficiency method for performance evaluation, European Journal of Operational Research, 280(1), 219-229.

Chiang Lee, Y. (2019). Ranking DMUs by Combining Cross-Efficiency Scores Based on Shannon's Entropy, Entropy, 21(5), 467.

Davtalab-Olyaie, M. A. (2019). Secondary goal in DEA cross-efficiency evaluation: A one home run is much better than two doubles criterion, Journal of the Operational Research Society, 70(5), 807-816.

Ehrgott, M. (2000). Multicriteria Optimization, Lecture Notes in Economics and Mathematical Systems, 491. Springer-Verlag, Berlin.

Emrouznejad. A. \& Liang Yang, G. (2018). A survey and analysis of the first 40 years of scholarly literature in DEA: 1978-2016, Socio-Economic Planning Sciences, 61, 4-8.

Ho, Q., Wang, M. \& Zhou, Q. (2018). Improved DEA Cross Efficiency Evaluation Method Based on Ideal and Anti-Ideal Points, Discrete Dynamics in Nature and Society, 1-9.

Hosseinzadeh Lotfi, F., Navabakhsh,M., Tehranian, A. \& Rostamy-Malkhalifeh, M. (2007). Ranking bank branches with interval data the application of DEA, International Mathematical Forum, 9(2), 429-440.
Karimi, B., Davtalab-Olyaie, M. \& Abdali, A. A. (2018). A Suitable Business Model for Bank Branches: Combining Business Model and Malmquist Productivity Index (MPI), Business Economic Journal, 9(2), 348.

Liang, L., Wu, J., Cook, W. D. \& Zhu, J. (2008). Alternative secondary goals in DEA cross-efficiency evaluation, International Journal of Production Economics, 113(2), 1025-1030.

Lotfi, M. R. \& Rostamy-MaleKhalifeh, M. (2016). Secondary Model Developed for Weight Selective in Evaluating the Efficiency of Cross-DEA with Fuzzy Data. International Journal of Data Envelopment Analysis, 4(3), 1031-1038.

Moeini, M., Karimi, B. \& Khorram, E. (2015). A Cross-Efficiency Approach for Evaluating Decision Making Units in Presence of Undesirable Outputs. In Modelling, Computation and Optimization in Information Systems and Management Sciences, 487498. Springer.

Oukil, A. \& Amin, G. R. (2015). Maximum appreciative cross-efficiency in DEA: A new ranking method, Computers \& Industrial Engineering, 81, 14-21.

Ramirez-Reyna, M. A., Cru-Pérez, F. A., CastellanosLopez, S. L., Hernandez-Valdez, G. \& RiveroAngeles, M. E. (2018). Differentiated Connection Admission Control Strategy for Wireless VoIP Networks with Adaptive Modulation Coding. In International Conference on Computing, Networking and Communications (ICNC), (pp. 31-37). IEEE. DOI:10.1109/ICCN.2018.8390322

Rodder, W. \& Reucher, E. (2001). A consensual peerbased DEA-model with optimized cross-efficiencies: input allocation instead of radial reduction, European Journal of Operational Research, 212(1), 148-154.

Sexton, T. R., Silkman, R. H. \& Hogan, A. J. (1986). Data envelopment analysis: Critique and extensions. In Silkman, R. H. (ed.) Measuring Efficiency: An Assessment of Data Envelopment Analysis, 3, 73-105.

Wu, J., Chu, J., Zhu, Q., Yin, P. \& Liang. L. (2016). DEA Cross-efficiency Evaluation Based on Satisfaction Degree: An Application to Technology Selection, International Journal of Production Research, 54(20), 5990-6007.

Zanuttini, D., Andres, C., Gonzalez, J., Lacapmesure, A., Priano, D., Nicolás, P., Pucci, N. \& Bullian, P. (2018). Using Software Defined Networking for Call Admission Control and VoIP applications. In 2018 Congreso Argentino de Ciencias de la Informática y Desarrollos de Investigación (CACIDI), (pp. 1-5). DOI: 10.1109/CACIDI.2018.8584371

Zhou, X., Xu, Z., Chai, J., Yao, L., Wang, S. \& Lev, B. (2018). Efficiency evaluation for banking systems under uncertainty: A multi-period three-stage DEA model, Omega, 85, 68-62. 